\title{
Minimal surfaces with mixed three-form flux
}

\author{
Rafael Hernández, Juan Miguel Nieto, ${ }^{\dagger}$ and Roberto Ruiz \\ Departamento de Física Teórica Universidad Complutense de Madrid 28040 Madrid, Spain
}

(Received 12 December 2018; published 5 April 2019)

\begin{abstract}
We study minimal area world sheets ending on two concentric circumferences on the boundary of Euclidean $\mathrm{AdS}_{3}$ with mixed Ramond-Ramond and Neveu-Schwarz-Neveu-Schwarz (NS-NS) three-form fluxes. We solve the problem by reducing the system to a one-dimensional integrable model. We find that the NS-NS flux term either brings the surface near to the boundary or separates the circumferences. In the limit of pure NS-NS flux, the solution adheres to the boundary in the former case, and the outer radius diverges in the latter. We further construct the underlying elliptic spectral curve, which allows us to analyze the deformation of other related minimal surfaces. We show that in the regime of pure NS-NS flux the elliptic curve degenerates.
\end{abstract}

DOI: 10.1103/PhysRevD.99.086003

\section{INTRODUCTION}

The AdS/CFT correspondence states that the strong coupling limit of the expectation value of Wilson loop observables can be described by classical string solutions. In particular, it is given by the area of the minimal surface swept by a string subtending the path determined by the loop on the boundary of the anti-de Sitter space [1]. A general class of minimal surfaces was found in Ref. [2] using a periodic ansatz that allowed the authors to reduce the analysis of the nonlinear sigma model for the string to the construction of solutions to one-dimensional integrable systems. This periodic ansatz is closely related to the ansatz introduced in Ref. [3] to transform the analysis of the energy spectrum of circular strings spinning in $\mathrm{AdS}_{5} \times S^{5}$ into the study of periodic solutions to the Neumann-Rosochatius integrable system, which is a model of oscillators on a sphere. In Ref. [4], it was shown that the analysis of closed strings spinning in $\mathrm{AdS}_{3} \times S^{3} \times T^{4}$ with a mixture of RamondRamond (R-R) and Neveu-Schwarz-Neveu-Schwarz (NS-NS) three-form fluxes can be performed by means of an integrable deformation of the Neumann-Rosochatius mechanical system (see Refs. [5-31] for other solutions and various problems regarding the integrability of the $\mathrm{AdS}_{3} / \mathrm{CFT}_{2}$ correspondence with mixed fluxes). The purpose of the present article is to extend the approach of

\footnotetext{
rafael.hernandez@fis.ucm.es

juanieto@ucm.es

roruiz@ucm.es
}

Published by the American Physical Society under the terms of the Creative Commons Attribution 4.0 International license. Further distribution of this work must maintain attribution to the author(s) and the published article's title, journal citation, and DOI. Funded by SCOAP .
Ref. [2] to find minimal area surfaces ending on two concentric circumferences in the presence of NS-NS flux using the integrable deformation of their associated mechanical system.

Besides the study of some aspects of the AdS/CFT correspondence in terms of simple mechanical models, the integrability of the problem has also lead to several complementary series of developments. One of them comes from the use of algebraic spectral curves to encode the dynamics of spinning string solutions, as was first noted in Ref. [32]. The construction of the spectral curves starts from the existence of nontrivial holonomies on the world sheet of the closed string. Therefore, when trying to construct algebraic curves for or world sheets ending on loops, one should face the problem that the holonomies may be trivial therein. However, factorizability of some classes of solutions allowed us to circumvent the absence of nontrivial holonomies since the construction just requires the local form of the Lax connection whenever it is present $[33,34]$. As our minimal solution with mixed flux preserves factorizability, we will follow this path here and adapt the procedure applied in Ref. [35] to the case of a nonvanishing NS-NS three-form.

The plan of the remaining part of the paper is as follows. In Sec. II, we will employ the integrability of the problem to construct minimal area surfaces ending on an annulus with mixed R-R and NS-NS fluxes. We will show that the presence of the NS-NS flux term either reduces the distance that the string is allowed to enter into the bulk or increases the separation between the circumferences on the boundary. In the limit of pure NS-NS flux, the action reduces to a supersymmetric Wess-Zumino-Witten model. We will prove that in this limit the world sheet is stuck on the boundary in the former case and that the radius of the outer circumference diverges in the latter. We will also discuss the existence of 
D1-string solutions related to these surfaces. In Sec. III, we will tackle the problem by constructing the associated spectral curve. We will use this result to recover the spectral curves of other configurations in some particular limits. We will find the deformation of the underlying elliptic surface by the flux term and show that either one or both cycles of the complex torus degenerate when the R-R flux vanishes. In Sec. IV, we summarize our results and comment on some open issues and future perspectives.

\section{MINIMAL AREA SURFACES}

In this section, we will construct the classical string solutions corresponding to a minimal surface that ends on two concentric circumferences on the boundary of the antide Sitter space in the presence of flux. In particular, we will be interested in classical string solutions embedded in Euclidean $\mathrm{AdS}_{3}$ with nonvanishing NS-NS three-form flux. We will write the background metric as

$$
\mathrm{d} s^{2}=z^{-2}\left(\mathrm{~d} r^{2}+r^{2} \mathrm{~d} \theta^{2}+d z^{2}\right)
$$

and the NS-NS B-field as

$$
B=i q z^{-2} r \mathrm{~d} r \wedge \mathrm{d} \theta
$$

where $|q| \leq 1$. The case where $q=0$ corresponds to the limit of pure R-R flux, while, setting $|q|=1$, we are left with pure NS-NS flux. In the above coordinates, the surface ending on the annulus is described by the ansatz [2] ${ }^{1}$

$$
r(\tau, \sigma)=r(\tau), \quad \theta(\tau, \sigma)=k \sigma, \quad z(\tau, \sigma)=z(\tau),
$$

together with the boundary conditions

$$
r\left(\tau_{i}, \sigma\right)=R_{i}, \quad \theta\left(\tau_{i}, \sigma\right)=k \sigma, \quad z\left(\tau_{i}, \sigma\right)=0, \quad i=1,2,
$$

with $R_{i}$ the radii of the circles and $k$ the winding index along the circumferences. When we enter this ansatz into the Euclidean world sheet action in the conformal gauge, we find a one-dimensional integrable mechanical model with Lagrangian

$$
L=z^{-2}\left(\dot{r}^{2}+\dot{z}^{2}-2 q k r \dot{r}+r^{2} k^{2}\right) .
$$

The integrability of this model follows from the existence of a first integral of motion associated to the dilatation invariance of the action. This integral parametrizes the solution and reads

\footnotetext{
${ }^{1}$ Here and throughout this article, $\tau$ denotes the Euclidean world sheet time coordinate.
}

$$
p=z^{-2}\left(r \dot{r}+z \dot{z}-q k r^{2}\right) .
$$

In order to solve this equation, we will also need the vanishing-energy constraint imposed by the conformal gauge condition,

$$
\dot{r}^{2}+\dot{z}^{2}-k^{2} r^{2}=0
$$

For convenience, we will introduce new variables $u$ and $v$, defined through

$$
z=\frac{u e^{v}}{\sqrt{1+u^{2}}}, \quad r=\frac{e^{v}}{\sqrt{1+u^{2}}} .
$$

In these coordinates, Eqs. (2.6) and (2.7) become

$$
\begin{gathered}
\dot{u}^{2}=\left(1-q^{2}\right) k^{2}+\left(k^{2}-2 q k p\right) u^{2}-p^{2} u^{4}, \\
\dot{v}=\frac{u^{2} p+q k}{1+u^{2}},
\end{gathered}
$$

while the boundary conditions read

$$
u\left(\tau_{i}\right)=0, \quad v\left(\tau_{i}\right)=\log R_{i}, \quad i=1,2 .
$$

The problem is therefore reduced to finding the solution to the differential equation (2.9). This equation defines a genus-1 complex surface, and thus the solution can be written in terms of elliptic functions.

In order to find the solutions to (2.9), we will separate the cases with $|p|>0$ and $p=0$. We will consider first the case with $|p|>0$. Equation (2.9) can be then rewritten as

$$
\dot{u}^{2}=p^{2}\left(u_{-}^{2}+u^{2}\right)\left(u_{+}^{2}-u^{2}\right)=P_{4}(u),
$$

with squared roots

$u_{ \pm}^{2}=\frac{ \pm\left(k^{2}-2 q k p\right)+\sqrt{\left(k^{2}-2 q k p\right)^{2}+4\left(1-q^{2}\right) k^{2} p^{2}}}{2 p^{2}}$.

These squared roots satisfy $u_{ \pm}^{2} \geq 0$. In the limit where $q= \pm 1$, the equality is reached by $u_{+}^{2}$ if we choose $k^{2} \mp 2 k p \leq 0$ or by $u_{-}^{2}$ if $k^{2} \mp 2 k p \geq 0$. Since $q= \pm 1$ corresponds to the situation where two of the roots in the polynomial $P_{4}(u)$ coalesce, one of the cycles of the complex surface degenerates in this limit.

The coordinate $u$ varies from the boundary at $u=0$ to a maximum value $u_{+}$corresponding to the turning point where $\dot{u}=0$. This is the branch of the solution with $\dot{u}>0$. Beyond the turning point, we have to move to the branch that extends from $u_{+}$to the boundary, where $\dot{u}<0$. We choose the interval of integration to go from $\tau_{1}=0$ to $\tau_{2}=T$ so that the turning point is located at $\tau=T / 2$. If we define 
$\alpha(u) \equiv \arcsin _{\left[0, \frac{\pi}{2}\right]}\left(\sqrt{\frac{u_{-}^{2}+u_{+}^{2}}{u_{-}^{2}+u^{2}}} \frac{u}{u_{+}}\right), \quad \kappa \equiv \frac{u_{+}}{\sqrt{u_{-}^{2}+u_{+}^{2}}}$,

the solution to Eq. (2.12) can be expressed as

$$
\begin{gathered}
\tau=\frac{F(\alpha, \kappa)}{|p| \sqrt{u_{-}^{2}+u_{+}^{2}}}, \quad \text { when } 0 \leq \tau \leq \frac{T}{2}, \\
\tau=\frac{2 K(\kappa)-F(\alpha, \kappa)}{|p| \sqrt{u_{-}^{2}+u_{+}^{2}}}, \quad \text { when } \frac{T}{2} \leq \tau \leq T,
\end{gathered}
$$

where $F(\alpha, \kappa)$ and $K(\kappa)$ are, respectively, the incomplete and complete elliptic integrals of the first kind. Therefore, the length of the interval of integration is given by

$$
T=\frac{2 K(\kappa)}{|p| \sqrt{u_{-}^{2}+u_{+}^{2}}}
$$

Inverting relations (2.15) and (2.16), we conclude that $u^{2}(\tau)=\frac{u_{-}^{2} u_{+}^{2}}{u_{-}^{2}+u_{+}^{2}} \operatorname{sd}^{2}\left(|p| \sqrt{u_{-}^{2}+u_{+}^{2}} \tau, \kappa\right)$, with $0 \leq \tau \leq T$,

where we have used the parity and semiperiodicity properties of the Jacobi function

$\operatorname{sd}(-z, \kappa)=-\operatorname{sd}(z, \kappa), \quad \operatorname{sd}(z+2 K(\kappa), \kappa)=-\operatorname{sd}(z, \kappa)$.

We can now integrate Eq. (2.10) to find

$$
\begin{aligned}
v(\tau)= & \frac{u_{-}^{2}(p-q k) \Pi\left(\operatorname{am}\left(|p| \sqrt{u_{-}^{2}+u_{+}^{2}} \tau, \kappa\right), n, \kappa\right)}{|p|\left(1-u_{-}^{2}\right) \sqrt{u_{-}^{2}+u_{+}^{2}}} \\
& -\frac{u_{-}^{2} p-q k}{1-u_{-}^{2}} \tau+\log R_{1},
\end{aligned}
$$

where $\Pi(\alpha, n, \kappa)$ is the incomplete elliptic integral of the third kind, with characteristic

$$
n=\frac{u_{+}^{2}\left(1-u_{-}^{2}\right)}{u_{-}^{2}+u_{+}^{2}} .
$$

The ratio of the radii $R=R_{2} / R_{1}$ can be obtained by setting $\tau=T$ in (2.20),

$$
R=\exp \left(\frac{2 u_{-}^{2}(p-q k) \Pi(n, \kappa)-2\left(u_{-}^{2} p-q k\right) K(\kappa)}{|p|\left(1-u_{-}^{2}\right) \sqrt{u_{-}^{2}+u_{+}^{2}}}\right),
$$

where we have used (2.17) and $\Pi(n, \kappa)$ is the complete elliptic integral of the third kind.

We will consider now the case of pure NS-NS flux, where $q=1$ (the limit where $q=-1$ can be obtained from the $q=1$ case by performing the substitution $k \rightarrow-k$ ). We must first note that the solution (2.18) displays sharply different behaviors depending on the sign of $k^{2}-2 k p$. In the region where $k^{2}-2 k p<0$, we find

$$
u_{+}^{2} \rightarrow 0, \quad u_{-}^{2} \rightarrow-\frac{k^{2}-2 k p}{p^{2}} .
$$

According to (2.18), the world sheet penetrates into the bulk from the boundary at $u=0$ until it reaches $u=u_{+}$. As the flux grows, the position of the turning point $u_{+}$ decreases. In the limit of pure NS-NS flux, the world sheet adheres entirely to the boundary. Moreover, both the elliptic modulus $\kappa$ and the elliptic characteristic $n$ vanish. Therefore, it is straightforward to show that the length of the interval $T$ tends to ${ }^{2}$

$$
\bar{T}=\frac{\pi}{\sqrt{\left|k^{2}-2 k p\right|}},
$$

and the ratio $R$ of the radii tends to

$$
\bar{R}=\exp \left(\frac{k \pi}{\sqrt{\left|k^{2}-2 k p\right|}}\right)=\exp (k \bar{T}) .
$$

On the contrary, if $k^{2}-2 k p>0$, we find

$$
u_{+}^{2} \rightarrow \frac{k^{2}-2 k p}{p^{2}}, \quad u_{-}^{2} \rightarrow 0 .
$$

In this case, the elliptic modulus $\kappa$ tends to 1 , and from (2.17), we thus conclude that the integration length goes to infinity. In order to understand in more detail this case, it is convenient to shift the world sheet time coordinate through

$$
\tau \rightarrow \tau+\frac{K(\kappa)}{|p| \sqrt{u_{-}^{2}+u_{+}^{2}}},
$$

which corresponds to choosing its range from $-T / 2$ to $T / 2$, before applying the limit to Eq. (2.18). After performing the shift, we find

$u^{2}(\tau)=u_{+}^{2} \operatorname{cn}^{2}\left(|p| \sqrt{u_{-}^{2}+u_{+}^{2}} \tau, \kappa\right), \quad$ with $-\frac{T}{2} \leq \tau \leq \frac{T}{2}$,

and thus

\footnotetext{
${ }^{2}$ Subsequently, barred quantities are evaluated in the limit of pure NS-NS flux.
} 
$u^{2}(\tau) \rightarrow u_{+}^{2} \operatorname{sech}^{2}\left(\sqrt{k^{2}-2 k p \tau}\right), \quad$ with $-\infty<\tau<\infty$.

From Eq. (2.22), we conclude that $R$ diverges if $k$ is positive and that it goes to zero if $k$ is negative. In fact, asymptotically, it is satisfied,

$$
\bar{R} \simeq \exp (k \bar{T})
$$

as in the case where $k^{2}-2 k p \leq 0$. Finally, in the threshold case where $k^{2}-2 k p=0$, we find that $u_{ \pm}^{2} \rightarrow 0$, which corresponds to a world sheet stuck on the boundary that subtends an annulus for which the ratio of the radii either diverges or tends to zero.

We will now move to the case where $p=0$, which is a particular limit of the general solution presented above. Accordingly, the elliptic surface associated to (2.12) reduces its genus by 1. Moreover, it follows from Eq. (2.22) that in the limit of pure R-R flux the two radii coincide, and we are led to a circle on the boundary. Either applying the limit to (2.18) and (2.20) or starting from (2.9) and (2.10) and substituting $p=0$ therein, we find that

$$
\begin{gathered}
u(\tau)=\sqrt{1-q^{2}} \sinh (|k| \tau), \\
v(\tau)=\operatorname{arctanh}(q \tanh (k \tau))+\log R_{1} .
\end{gathered}
$$

If $q= \pm 1$, the world sheet adheres to the boundary, and the radius of the external circumference diverges. This fact is consistent with the previous discussion on the pure NS-NS flux limit, since $k^{2}>0$ is always satisfied.

We must note that Eqs. (2.10) and (2.12) with the boundary conditions (2.4) admit an infinite class of solutions that corresponds to a world sheet stuck on the boundary and which are absent everywhere but in the pure NS-NS flux limit. Integrating (2.10) with $u=0$ leads to $v=k \tau$. It follows then that the ratio of the radii and the length of the interval are related through $\bar{R}=\exp (k \bar{T})$. Given the winding index $k$, any value of $\bar{R}$ can be achieved for these solutions by choosing properly the length $\bar{T}$ and vice versa.

We will conclude this section with a brief discussion on the limiting cases of pure R-R and NS-NS flux by means of the $S L(2, \mathbb{Z})$ symmetry of type IIB strings [36]. Let us first note that, since $\mathrm{AdS}_{3} \times S^{3} \times T^{4}$ supported by mixed flux emerges as the near horizon limit of a brane array [37], it does not need to respect the full $S L(2, \mathbb{Z})$ symmetry. Therefore, we have to limit ourselves to those transformations that do not modify its field content. In particular, given that both the dilaton and the R-R scalar vanish initially [6], they must also vanish after performing the $S L(2, \mathbb{Z})$ transformation. If we take into account the properties of the fields, we realize that just trivial and S-duality transformations are allowed [38]. Since S-duality interchanges F1- and D1-string numbers, we conclude that the F1-strings that we have found with vanishing R-R flux imply the existence of D1-strings with vanishing NS-NS flux which display a behavior analogous to the former and vice versa. We may also draw this conclusion starting from the Dirac-Born-Infeld action with a Wess-Zumino term for the R-R two-form. The Euler-Lagrange equations for the gauge potential imply that the world sheet Abelian electric field $\mathcal{F}=B+2 \pi \alpha^{\prime} F$ is related to a constant field and that it vanishes when the latter does [38]. Furthermore, the boundary conditions on the spatial world sheet coordinate imply that the constant field is semiclassically quantized in terms of the F1-string number of the solution [39], which is zero for D1-strings. Since $\mathcal{F}$ vanishes, we could apply the second order formalism in the action. Therefore, the ansatz (2.3) with the periodic boundary conditions (2.4) would have led us to the Lagrangian (2.5) with the NS-NS flux term replaced by the one for the R-R flux, i.e., with $q$ replaced by $\pm \sqrt{1-q^{2}}$.

\section{ALGEBRAIC CURVES AND MINIMAL SURFACES}

In this section, we will extend the analysis of the case of pure R-R flux in Ref. [35] to construct the deformation by the NS-NS flux term of the algebraic elliptic curve that describes the minimal surfaces ending on an annulus on the boundary of Euclidean $\mathrm{AdS}_{3}$ that we have obtained above. Under the presence of mixed R-R and NS-NS fluxes, the Lax connection is deformed [5,11], and therefore the spectral curve defined by the quasimomenta [11,33,34] should also be deformed. In order to find this deformation, we will start by fixing the gauge condition for the coset group element to $g_{L} \oplus g_{R}=1 \oplus g$ and then performing a Wick rotation both in the space-time and the world sheet coordinates. We will choose the group element for Euclidean $\mathrm{AdS}_{3}$ as

$$
g=S^{-1} \frac{1}{z}\left(\begin{array}{cc}
r^{2}+z^{2} & r \\
r & 1
\end{array}\right) S
$$

where $S=e^{-i(\theta / 2) \sigma_{3}}$. When we take into account the ansatz (2.3), the parametrization of the group element becomes factorizable, i.e., $g(\tau, \sigma)=S^{-1}(\sigma) g(\tau, 0) S(\sigma)$. This property is inherited by the Maurer-Cartan form $j=g^{-1} \mathrm{~d} g$, the components of which read

$$
\begin{gathered}
j_{\tau}=S^{-1} \frac{1}{z^{2}}\left(\begin{array}{cc}
r \dot{r}+z \dot{z} & \dot{r} \\
\left(z^{2}-r^{2}\right) \dot{r}-2 r z \dot{z} & -(r \dot{r}+z \dot{z})
\end{array}\right) S, \\
j_{\sigma}=S^{-1} \frac{i k}{z^{2}}\left(\begin{array}{cc}
r^{2} & r \\
-\left(r^{2}+z^{2}\right) r & -r^{2}
\end{array}\right) S .
\end{gathered}
$$


The components of the relevant part of the deformed Lax connection are [11]

$$
A_{\tau}=\frac{(q x+\tilde{q}) j_{\tau}+i x j_{\sigma}}{\tilde{q}(s-x)(1 / s+x)}, \quad A_{\sigma}=\frac{(q x+\tilde{q}) j_{\sigma}-i x j_{\tau}}{\tilde{q}(s-x)(1 / s+x)},
$$

where $x$ denotes the spectral parameter, and we have introduced $s=\sqrt{(1-q) /(1+q)}$ and $\tilde{q}=\sqrt{1-q^{2}} .3$ We must stress that the representation of the classical solution in terms of a complex surface does not require the explicit form of the monodromy matrix because $S^{-1} \mathrm{~d} S=$ $-i(k / 2) \sigma_{3} \mathrm{~d} \sigma$ [34]. It is just the local form of the Lax connection that is implied. If we define

$$
L(\tau, x)=A_{\sigma}(\tau, \sigma=0, x)+i \frac{k}{2} \sigma_{3},
$$

the quasimomenta $\pm p(x)$ that define the complex surface read [35]

$$
p(x)=2 \pi \sqrt{\operatorname{det} L(\tau, x)}+k \pi .
$$

Using now Eqs. (3.2)-(3.5), we immediately conclude that

$$
\begin{aligned}
\operatorname{det} L= & \frac{1}{4 \tilde{q}^{2}(s-x)^{2}(1 / s+x)^{2} z^{2}}\left[4 x^{2}\left(\dot{r}^{2}+\dot{z}^{2}\right)\right. \\
& -4 k x(r \dot{r}+z \dot{z})\left[\tilde{q}\left(1-x^{2}\right)+2 x q\right] \\
& +4 k^{2} x(\tilde{q}+q x)(q-\tilde{q} x) r^{2} \\
& \left.+k^{2}\left[\tilde{q}\left(1-x^{2}\right)+2 x q\right]^{2} z^{2}\right]
\end{aligned}
$$

that can be rewritten as

$$
\begin{aligned}
\operatorname{det} L= & \frac{k}{4 \tilde{q}^{2}(s-x)^{2}(1 / s+x)^{2}}\left[k \tilde{q}^{2} x^{4}+4(p-q k) \tilde{q} x^{3}\right. \\
& \left.+\left(6 q^{2} k-2 k-8 q p\right) x^{2}-4(p-q k) \tilde{q} x+k \tilde{q}^{2}\right]
\end{aligned}
$$

where we have made use of relations (2.6) and (2.7). We must note that the time dependence is lost as ensured by the isospectral property of the spectrum of the monodromy matrix. In the limit of pure NS-NS flux, the quasimomenta trivializes abruptly,

$$
\bar{p}=2 \pi \sqrt{k^{2}-2 p k}+k \pi,
$$

becoming independent of the spectral parameter. Note that the sign of the combination $k^{2}-2 k p$, which as we have

\footnotetext{
${ }^{3}$ Note that integrability just implies the condition $q^{2}+\tilde{q}^{2}=1$ [5]. However, for concreteness, it will be assumed throughout the subsequent discussion that $0 \leq q, \tilde{q} \leq 1$.
}

discussed in the previous section governs the behavior of the solution in the limit $q=1$, may be regarded as a reality condition on the shifted quasimomenta $\bar{p}-k \pi$.

The equation of the algebraic curve defined by the quasimomenta can be constructed from (3.8) by setting aside the poles of the Lax connection [34],

$$
\operatorname{det}\left[y^{2}-(s-x)(1 / s+x) L\right]=0,
$$

which leads to

$$
\begin{aligned}
y^{2}= & \frac{k^{2}}{4} x^{4}+\frac{k(p-q k)}{\tilde{q}} x^{3}+\frac{k\left(3 q^{2} k-k-4 q p\right)}{2 \tilde{q}^{2}} x^{2} \\
& -\frac{k(p-q k)}{\tilde{q}} x+\frac{k^{2}}{4} .
\end{aligned}
$$

In order to understand the behavior of this algebraic curve in the limits of interest, we will redefine $x$ and $y$ to bring the previous equation into the Weierstrass form (see, for instance, Ref. [40]),

$$
y^{2}=4 x^{3}-g_{2} x-g_{3},
$$

where the functions $g_{2}$ and $g_{3}$ are given by

$$
\begin{aligned}
g_{2}= & \frac{64}{3}\left[\left(1-3 q^{2}+3 q^{4}\right) k^{4}+2 p q\left(1-3 q^{2}\right) k^{3}\right. \\
& \left.+q^{2}\left(4 p^{2}+3-3 q^{2}\right) k^{2}-3 \tilde{q}^{2}\left(q p k-p^{2}\right)\right], \\
g_{3}= & \frac{256}{27} k(k-2 p q)\left[\left(2-9 q^{2}+9 q^{4}\right) k^{4}\right. \\
& +2 p q\left(5-9 q^{2}\right) k^{3}+q^{2}\left(8 p^{2}+9-9 q^{2}\right) k^{2} \\
& \left.-9 \tilde{q}^{2}\left(2 q p k-p^{2}\right)\right] .
\end{aligned}
$$

The $j$-invariant of the algebraic curve can then be found through

$$
j=\frac{1728 g_{2}^{3}}{\Delta},
$$

where the modular discriminant $\Delta=g_{2}^{3}-27 g_{3}^{2}$ is

$$
\begin{aligned}
\Delta= & 27 \tilde{q}^{4}\left[k^{4}+4 p^{2}+4 q p k\left(k^{2}-2\right)\right. \\
& \left.+4 q^{2}\left(1-k^{2}\right)\left(\tilde{q}^{2} k^{2}-2 k p q-p^{2}\right)\right] \\
& \times\left[p^{2}+q k\left(1-k^{2}\right)(q k-2 p)\right]^{2} .
\end{aligned}
$$

The $j$-invariant depends on the modular parameter $\omega=$ $\omega_{2} / \omega_{1}$, with $\omega_{1}$ and $\omega_{2}$ the periods of the complex torus. This function is invariant under modular transformations and discriminates between elliptic curves over the complex numbers belonging to different isomorphism classes.

In the case where $q=0$ and $k= \pm 1$, the algebraic curve (3.12) describes other minimal surfaces in some limiting cases, namely, the world sheet subtending a circle when 
$p=0$ and two parallel lines when $p$ tends to infinity. From the point of view of the $j$-invariant, the circle corresponds to $j=\infty$, where one of the cycles of the torus collapses, and the two parallel lines correspond to $j=1728$, where the group of automorphisms of the curve is enhanced. The solution corresponding to the last case is obtained directly from ours by making the two radii tend to infinity while keeping their radial distance finite [35].

To obtain the extension of the circle under NS-NS flux, we will set $k= \pm 1$ and solve $1 / j=0$ for $p$. If we impose that $p=0$ when $q=0$, we still have $p=0$ as a solution for any value of the flux. The algebraic curve then reads

$$
y^{2}=4 x^{3}-\frac{64}{3} x-\frac{512}{27},
$$

which is not deformed by the flux term. We stress, on the basis of the discussion above (2.31) and (2.32) and the analysis performed here, that the most natural extension of the circle with nonvanishing NS-NS flux involves its transformation into an annulus at the boundary. In the two parallel lines case, we can proceed analogously. If we set $k= \pm 1$ and solve $j=1728$ for $p$ requiring that $p \rightarrow \infty$ when $q=0$, we find that $p= \pm 1 / 2 q$. The algebraic curve in the Weierstrass form now reads

$$
y^{2}=4 x^{3}-16 \frac{1-q^{2}}{q^{2}} x .
$$

Note that the value of $p$ in the limit of pure NS-NS flux is that of the threshold $k^{2}-2 k p=0$, with $k= \pm 1$. We should also mention that other solutions, with different values for $p$ and absent in the pure $\mathrm{R}-\mathrm{R}$ regime and that share the same $j$-invariant with the ones considered above, can be obtained by allowing other values for $k$ and loosening the condition on $p$ when $q=0$.

Let us focus now on the limit of pure NS-NS flux. In this case, $g_{2}$ and $g_{3}$ become

$\bar{g}_{2}=\frac{64}{3}\left(k^{2}-2 k p\right)^{2}, \quad \bar{g}_{3}=\frac{512}{27}\left(k^{2}-2 k p\right)^{3}$,

and the elliptic discriminant vanishes. In terms of the roots $e_{i}$ of (3.12), we get

$$
\begin{aligned}
& \bar{e}_{1}=-\frac{2}{3}\left(k^{2}-2 k p\right)+2\left|k^{2}-2 k p\right|, \\
& \bar{e}_{2}=-\frac{2}{3}\left(k^{2}-2 k p\right)-2\left|k^{2}-2 k p\right|, \\
& \bar{e}_{3}=-\frac{4}{3}\left(k^{2}-2 k p\right),
\end{aligned}
$$

and hence either $e_{1}$ or $e_{2}$ coalesces with $e_{3}$, depending on the sign of $k^{2}-2 k p$. The superposition of two roots, or equivalently the vanishing of the elliptic discriminant, implies a divergent $j$-invariant, which corresponds to the modular parameter $\bar{\omega}=i \infty$. Therefore, one of the cycles of the complex torus degenerates, and the latter gets pinched, becoming topologically equivalent to a complex sphere with two points identified. If the condition $k^{2}-2 k p=0$ is also satisfied, $\bar{g}_{2}=\bar{g}_{3}=0$, and the three roots coalesce to zero. The $j$-invariant is then $\bar{j}=1728$, which corresponds to $\bar{\omega}=i$. In the torus picture, the two points identified become the same when such a threshold is reached, and thus the pinched torus turns into a sphere.

\section{CONCLUSIONS}

In this article, we have constructed minimal surfaces that end on two concentric circumferences in Euclidean $\mathrm{AdS}_{3}$ with mixed R-R and NS-NS three-form fluxes. We have shown that the effect of the NS-NS flux term is either to decrease the distance that the minimal surface is allowed to enter into the bulk or to increase the relative radii of the circles at the boundary. By taking the limit of pure NS-NS flux, we have found that in the first case the string world sheet remains stuck on the boundary while in the second case the external radius diverges. We have also argued the existence of analogous classical D1-string solutions in the regime of pure R-R flux. We have further constructed the spectral curve that describes the solutions that we have found, studying various limiting cases that should describe the deformation under mixed flux of other relevant world sheets. Finally, we have found that the pure NS-NS limit of the solution may be regarded as a degenerate limit of its associated complex torus.

The family of minimal surfaces constructed here should be straightforwardly generalizable when nonvanishing angular momentum in the sphere is included. In particular, these solutions encompass the world sheet stretching between a circle and a point in the limit of pure R-R flux when one of the circumferences collapses. As the nontrivial mixture of fluxes would again deform these world sheets, their study may shed light on the limit of pure NS-NS flux of minimal surfaces, indicating if the behavior of the world sheet we have encountered persists. Moreover, this extension may open the possibility of finding other classes of solutions in the mixed flux regime that would be otherwise absent.

It would be also interesting to study if any phase transition occurs either for the minimal area surfaces that we have found or for their extension endowed with angular momentum in the sphere. Regarding the on-shell regularized action, the authors of Ref. [41] found a phase transition between connected and disconnected world sheets in $\mathrm{AdS}_{5} \times S^{5}$ depending on the axial distance between two concentric circumferences on the boundary. Performing an analogous analysis, it could be possible to establish which are the preferential solutions in terms of their action, including the different classes found here in the regime of pure NS-NS flux. Furthermore, it could happen that the flux mixing term might tune some possible phases of minimal surfaces. 


\section{ACKNOWLEDGMENTS}

The work of R. H. is supported by Grant No. FPA2014-54154-P and by Banco Santander Central Hispano-Universidad Complutense de Madrid (BSCH-UCM) through Grant No. GR3/14-A 910770.

[1] J. M. Maldacena, Wilson Loops in Large N Field Theories, Phys. Rev. Lett. 80, 4859 (1998); S. J. Rey and J. T. Yee, Macroscopic strings as heavy quarks in large $\mathrm{N}$ gauge theory and anti-de Sitter supergravity, Eur. Phys. J. C 22, 379 (2001).

[2] N. Drukker and B. Fiol, On the integrability of Wilson loops in $\mathrm{AdS}_{5} \times S^{5}$ : Some periodic ansatze, J. High Energy Phys. 01 (2006) 056.

[3] G. Arutyunov, S. Frolov, J. Russo, and A. A. Tseytlin, Spinning strings in $\mathrm{AdS}_{5} \times S^{5}$ and integrable systems, Nucl. Phys. B671, 3 (2003); G. Arutyunov, J. Russo, and A. A. Tseytlin, Spinning strings in $\mathrm{AdS}_{5} \times S^{5}$ : New integrable system relations, Phys. Rev. D 69, 086009 (2004).

[4] R. Hernández and J. M. Nieto, Spinning strings in $\mathrm{AdS}_{3} \times S^{3}$ with NS-NS flux, Nucl. Phys. B888, 236 (2014).

[5] A. Cagnazzo and K. Zarembo, B-field in $\mathrm{AdS}_{3} / C F T_{2}$ Correspondence and integrability, J. High Energy Phys. 11 (2012) 133; Erratum, J. High Energy Phys. 04 (2013) 3.

[6] B. Hoare and A. A. Tseytlin, On string theory on $\mathrm{AdS}_{3} \times$ $S^{3} \times T^{4}$ with mixed 3-form flux: Tree-level S-matrix, Nucl. Phys. B873, 682 (2013).

[7] B. Hoare and A. A. Tseytlin, Massive S-matrix of $\mathrm{AdS}_{3} \times$ $S^{3} \times T^{4}$ superstring theory with mixed 3-form flux, Nucl. Phys. B873, 395 (2013); B. Hoare, A. Stepanchuk, and A. A. Tseytlin, Giant magnon solution and dispersion relation in string theory in $\mathrm{AdS}_{3} \times S^{3} \times T^{4}$ with mixed flux, Nucl. Phys. B879, 318 (2014).

[8] C. Ahn and P. Bozhilov, String solutions in $\mathrm{AdS}_{3} \times S^{3} \times T^{4}$ with NS-NS B-field, Phys. Rev. D 90, 066010 (2014).

[9] J. R. David and A. Sadhukhan, Spinning strings and minimal surfaces in $\mathrm{AdS}_{3}$ with mixed 3-form fluxes, J. High Energy Phys. 10 (2014) 049.

[10] A. Banerjee, K. L. Panigrahi, and P. M. Pradhan, Spiky strings on $\mathrm{AdS}_{3} \times S^{3}$ with NS-NS flux, Phys. Rev. D 90 , 106006 (2014).

[11] A. Babichenko, A. Dekel, and O. Ohlsson Sax, Finite-gap equations for strings on $\mathrm{AdS}_{3} \times S^{3} \times T^{4}$ with mixed 3-form flux, J. High Energy Phys. 11 (2014) 122.

[12] L. Bianchi and B. Hoare, $\mathrm{AdS}_{3} \times S^{3} \times M^{4}$ string S-matrices from unitarity cuts, J. High Energy Phys. 08 (2014) 097.

[13] R. Roiban, P. Sundin, A. Tseytlin, and L. Wulff, The oneloop worldsheet S-matrix for the $\mathrm{AdS}_{n} \times S^{n} \times T^{10-2 n}$ superstring, J. High Energy Phys. 08 (2014) 160.

[14] T. Lloyd, O. Ohlsson Sax, A. Sfondrini, and B. Stefański, Jr., The complete worldsheet $\mathrm{S}$ matrix of superstrings on $\mathrm{AdS}_{3} \times S^{3} \times T^{4}$ with mixed three-form flux, Nucl. Phys. B891, 570 (2015).
[15] M. Heinze, G. Jorjadze, and L. Megrelidze, Isometry group orbit quantization of spinning strings in $\mathrm{AdS}_{3} \times \mathrm{S}^{3}$, J. Phys. A 48, 125401 (2015).

[16] P. Sundin and L. Wulff, One- and two-loop checks for the $\mathrm{AdS}_{3} \times S^{3} \times T^{4}$ superstring with mixed flux, J. Phys. A 48, 105402 (2015).

[17] A. Stepanchuk, String theory in $\mathrm{AdS}_{3} \times S^{3} \times T^{4}$ with mixed flux: semiclassical and 1-loop phase in the $S$-matrix, J. Phys. A 48, 195401 (2015).

[18] R. Hernández and J. M. Nieto, Elliptic solutions in the Neumann-Rosochatius system with mixed flux, Phys. Rev. D 91, 126006 (2015).

[19] R. Borsato, O. Ohlsson Sax, A. Sfondrini, and B. Stefański, The $\mathrm{AdS}_{3} \times \mathrm{S}^{3} \times \mathrm{S}^{3} \times \mathrm{S}^{1}$ worldsheet $\mathrm{S}$ matrix, J. Phys. A 48, 415401 (2015).

[20] A. Banerjee, K. L. Panigrahi, and M. Samal, A note on oscillating strings in $\mathrm{AdS}_{3} \times S^{3}$ with mixed three-form fluxes, J. High Energy Phys. 11 (2015) 133.

[21] A. Banerjee and A. Sadhukhan, Multi-spike strings in $\mathrm{AdS}_{3}$ with mixed three-form fluxes, J. High Energy Phys. 05 (2016) 083.

[22] A. Banerjee, S. Biswas, and R. R. Nayak, D1 string dynamics in curved backgrounds with fluxes, J. High Energy Phys. 04 (2016) 172.

[23] R. Borsato, O. Ohlsson Sax, A. Sfondrini, B. Stefański, A. Torrielli, and O. Ohlsson Sax, On the dressing factors, Bethe equations and Yangian symmetry of strings on $\mathrm{AdS}_{3} \times \mathrm{S}^{3} \times \mathrm{T}^{4}$, J. Phys. A 50, 024004 (2017).

[24] M. S. P. Barik, M. Khouchen, J. Kluson, and K. L. Panigrahi, $S L(2, Z)$ invariant rotating $(m, n)$ strings in $\mathrm{AdS}_{3} \times S^{3}$ with mixed flux, Eur. Phys. J. C 77, 298 (2017).

[25] S. P. Barik, K. L. Panigrahi, and M. Samal, Perturbations of pulsating strings, Eur. Phys. J. C 78, 882 (2018).

[26] A. Pittelli, Yangian Symmetry of String Theory on $\mathrm{AdS}_{3} \times S^{3} \times S^{3} \times S^{1}$ with Mixed 3-form Flux, Nucl. Phys. B935, 271 (2018).

[27] R. Hernández, J. M. Nieto, and R. Ruiz, Pulsating strings with mixed three-form flux, J. High Energy Phys. 04 (2018) 078.

[28] O. Ohlsson Sax and B. Stefański, Closed strings and moduli in $\mathrm{AdS}_{3} / \mathrm{CFT}_{2}$, J. High Energy Phys. 05 (2018) 101.

[29] J. M. Nieto and R. Ruiz, One-loop quantization of rigid spinning strings in $\mathrm{AdS}_{3} \times S^{3} \times T^{4}$ with mixed flux, J. High Energy Phys. 07 (2018) 141.

[30] A. Dei and A. Sfondrini, Integrable spin chain for stringy Wess-Zumino-Witten models, J. High Energy Phys. 07 (2018) 109.

[31] A. Banerjee, S. Biswas, and K. L. Panigrahi, On multi-spin classical strings with NS-NS flux, J. High Energy Phys. 08 (2018) 053. 
[32] V. A. Kazakov, A. Marshakov, J. A. Minahan, and K. Zarembo, Classical/quantum integrability in AdS/CFT, J. High Energy Phys. 05 (2004) 024.

[33] R. A. Janik and P. Laskos-Grabowski, Surprises in the AdS algebraic curve constructions: Wilson loops and correlation functions, Nucl. Phys. B861, 361 (2012).

[34] A. Dekel, Algebraic curves for factorized string solutions, J. High Energy Phys. 04 (2013) 119.

[35] A. Dekel and T. Klose, Correlation function of circular Wilson loops at strong coupling, J. High Energy Phys. 11 (2013) 117.

[36] N. A. Obers and B. Pioline, U duality and M theory, an algebraic approach, PoS corfu 98, 053 (1998); Lect. Notes Phys. 525, 424 (1999).
[37] A. Giveon, D. Kutasov, and N. Seiberg, Comments on string theory on $\mathrm{AdS}_{3}$, Adv. Theor. Math. Phys. 2, 733 (1998).

[38] J. Kluson, Integrability of a D1-brane on a group manifold with mixed three-form flux, Phys. Rev. D 93, 046003 (2016); $(m, n)$-String in $(p, q)$-string and $(p, q)$-five-brane background, Eur. Phys. J. C 76, 582 (2016).

[39] C. Bachas and M. Petropoulos, Anti-de Sitter D-branes, J. High Energy Phys. 02 (2001) 025.

[40] K. Chandrasekharan, Elliptic Functions (Springer-Verlag, Berlin, 1985).

[41] D. J. Gross and H. Ooguri, Aspects of large N gauge theory dynamics as seen by string theory, Phys. Rev. D 58, 106002 (1998). 